\title{
Age-Dependent Changes in Temperature Regulation - A Mini Review
}

\author{
Clark M. Blatteis \\ Department of Physiology, College of Medicine, University of Tennessee Health Sciences Center, \\ Memphis, Tenn., USA
}

\section{Key Words}

Elderly · Thermoregulation - Thermogenesis •

Thermoreceptors $\cdot$ Cutaneous circulation $\cdot$ Behavior

\begin{abstract}
It is now well recognized that the body temperature of older men and women is lower than that of younger people and that their tolerance of thermal extremes is more limited. The regulation of body temperature does not depend on a single organ, but rather involves almost all the systems of the body, i.e. systems not exclusively dedicated to thermoregulatory functions such as the cardiovascular and respiratory systems. Since these deteriorate naturally with advancing age, the decrement in their functions resonates throughout all the bodily processes, including those that control body temperature. To the extent that the age-related changes in some of these, e.g. in the musculoskeletal system, can be slowed, or even prevented, by certain measures, e.g. fitness training, so can the decrements in thermoregulatory functions. Some deficits, however, are unavoidable, e.g. structural skin changes and metabolic alterations. These impact directly on the ability of the elderly to maintain thermal homeostasis, particularly when challenged by ambient thermal extremes. Since the maintenance of a relatively stable, optimal core temperature is one of the body's most important activities, its very survival can be threatened by these disorders. The
\end{abstract}

present article describes the principal, age-associated changes in physiological functions that could affect the ability of seniors to maintain their body temperature when exposed to hot or cold environments.

Copyright $\odot 2011$ S. Karger AG, Basel

Numerous epidemiological studies have indicated that the internal body ('core') temperature of both healthy men and women over 60-65 years of age is generally lower than that of their younger adult counterparts. The actual differences reported vary, however, depending on the gender, the site of measurement (oral, rectal, axillary, tympanic), the measurement device (type of thermometer), the ambient temperature, the time of day or season of year, the subjects' lifestyles, and other variables; so much so, in fact, that some workers have concluded that the core temperature does not change with advancing age. This notwithstanding, the average difference between clinically healthy adult (ages 20-64) and geriatric (ages 65-95) male and female cohorts reported in the literature is approximately $0.4^{\circ} \mathrm{C} / 0.7^{\circ} \mathrm{F}$, a statistically significant difference but physiologically innocuous. On the other hand and of greater practical consequence, there is agreement that the elderly are at greater risk of hypo- and hyperthermia when exposed to a cold or warm environment, respectively. Indeed, it is well documented that the

\section{KARGER}

Fax +41613061234

E-Mail karger@karger.ch

www.karger.com
(C) 2011 S. Karger AG, Basel

0304-324X/12/0584-0289\$38.00/0

Accessible online at:

www.karger.com/ger
Clark M. Blatteis, $\mathrm{PhD}$

Department of Physiology, College of Medicine

University of Tennessee Health Sciences Center

894 Union Avenue, Memphis, TN 38163 (USA)

Tel. +1 901448 5845, E-Mail cblattei@ uthsc.edu 
incidence of morbidity and mortality is higher among the aged than the adult during extended cold and heat waves [1-3]. An understanding, therefore, of the mechanisms that regulate body temperature and of how aging alters them is relevant to the care, comfort and health of older people. Hence, the purpose of this mini-review is briefly to describe the currently known age-associated changes in the several physiological functions that normally combine to maintain core temperature at its optimal level in healthy seniors.

\section{Body Temperature: A Quick Refresher}

The core temperature is the quantitative measure of the amount of heat contained ('stored') in the body, evaluated, thanks to the availability of thermometers, in ${ }^{\circ} \mathrm{C}$ or ${ }^{\circ} \mathrm{F}$ rather than in calories, which are technically cumbersome to measure. The heat stored in the body represents at any time the balance between the heat gained by it (either actively [metabolically] such as by performance of physical exercise or passively such as by exposure to a warm environment) and that lost from its surface to the ambient environment. Heat is always actively gained by the body because the catabolism of the substrates it continuously consumes to maintain its vital processes is not $100 \%$ efficient, the energy that is not used specifically for these processes therefore being wasted as heat. Hence, when extra heat is needed, the body's principal source is its largest mass, the skeletal muscle mass, and the amount of heat it wastefully generates is the greater when muscles contract yet perform no useful work, i.e. when they shiver. The heat thus generated is transferred from the core via conduction and circulatory convection down its thermal gradient to the body's surface ('shell' [skin]), whence it is dissipated to the surroundings by conduction, convection, and radiation so long as the ambient environment is cooler than the skin. But when the environment is warmer than the skin, the thermal gradient is reversed and heat is gained by the body from the environment. In this case, the only other means available to the body to lose its heat is by the evaporation of sweat. It follows from the preceding that the efficacy of these responses is a function of the amount of heat, i.e. the flow of warm blood that actually reaches the skin. The control of skin blood flow is, therefore, of paramount importance to the maintenance of normal core temperature.

It is thus apparent that the maintenance of body temperature at rest in a thermoneutral environment as well as under thermally stressful conditions involves the in- terrelation and integration of virtually all the systems of the body, namely the cardiovascular, respiratory, renal, endocrinometabolic, musculoskeletal, and innate immune systems. They are furthermore augmented by behavioral responses that, in fact, often precede them. The activation of all occurs when alerting signals from thermosensors both inside and outside the body warn the brain, the controller of these functions, that the core temperature is at risk and that corrective measures must be initiated to prevent its untoward up or down deviation. Hence, both the peripheral and central nervous systems are also involved. It can, therefore, be anticipated that dysfunctions occurring with advancing age at any of these multiple levels could affect the responsiveness of the elderly to thermal stress.

\section{Cardiovascular System}

\section{Cutaneous Vasomotor Control}

In the context of thermoregulation, the cutaneous circulation constitutes a variable heat insulator immediately below the skin's surface. The proximity of skin blood flow to the surface determines its thickness, i.e. the insulative value of this barrier. Thus, the closer is this flow to the surface the shorter is the distance that the heat it contains is conducted down its thermal gradient to the surface and lost - a benefit in a warm environment. Conversely, the farther it is from this surface the longer is the distance it has to traverse, i.e. heat is conserved - a benefit in a cold environment. The path of this blood flow is, naturally, determined by the patency of relevant vessels, and this, in turn, is controlled by the autonomic nervous system and various endogenous mediators.

\section{Cold Exposure}

To wit, the lumen diameter of all blood vessels is predominantly determined by the discharge frequency of the sympathetic nerve fibers innervating the vascular smooth muscle (VSM) layer of the vessels. In the nonglabrous (hairy) skin of young adults, these fibers release norepinephrine (NE) and its co-transmitter, neuropeptide Y (NPY), which bind to their relevant receptors (adrenergic $\alpha_{1}$ and $\alpha_{2}$ and NPY Y ${ }_{1}$ ) on the VSM cell. Another sympathetic co-transmitter, adenosine triphosphate (ATP) and its receptor, $\mathrm{P}_{2 \mathrm{x}}$, may also be involved. The greater the sympathetic discharge rate the more are the VSM stimulated to contract, narrowing the lumen diameter and thereby restricting the blood flow through the affected cutaneous vessels. The functional effect of this 
vasoconstriction is to limit significantly the perfusion of the epidermis and dermis, thereby allowing the skin to cool toward the ambient temperature, reducing the thermal difference between the two, and therefore decreasing the loss of body heat - the desired benefit in a cold environment. This is, in fact, the reflex cutaneous vascular response to whole body cooling $[4,5]$. The routing of skin blood flow close to or away from the surface is controlled by the opening and closing, respectively, of arteriovenous anastomoses (AVA). These are particularly abundant in glabrous (acral) skin and richly innervated by sympathetic nerves.

In aged subjects, the cold-induced discharge rate of the sympathetic nerves to the cutaneous vasculature is significantly reduced and both the synthesis and release of $\mathrm{NE}$ are decreased [6,7]. The latter is presumptively due to a decrease (caused by the demonstrated increase in reactive oxygen species [ROS] with advancing age) of the bioavailability of an essential co-factor of tyrosine hydroxylase, the rate-limiting enzyme that catalyzes the initial step in the conversion of L-tyrosine to NE, axonal tetrahydrobiopterin $\left(\mathrm{BH}_{4}\right)$. Moreover, the synthesis and release of NPY and ATP are essentially abolished and the population, hence the sensitivity, of the $\mathrm{Y}_{1}$ and $\mathrm{P}_{2 \mathrm{x}}$ receptors is reduced, resulting in the virtually complete loss of sympathetic co-transmitter function [7]. Consequently, the efficacy of cutaneous vasoconstriction is diminished in the elderly and their body's ability to retain heat in the cold is reduced, causing their core temperature to fall and putting them at risk of hypothermia.

A somewhat different vasoconstrictor mechanism operates upon localized skin cooling. Initially, lumen narrowing is mediated by $\mathrm{NE}$ already present locally from basal sympathetic activity, binding to $\alpha_{2}$-adrenoceptors. This action induces an increase in intracellular $\left[\mathrm{Ca}^{2+}\right]$, the consequent phosphorylation of myosin light chain (MLC) by MLC kinase (MLCK) and, ultimately, VSM contraction. This process is enhanced by an increase in the number of $\alpha_{2}$ adrenoceptors by their translocation from intracellular stores to the cell membrane. Subsequently, nonadrenergic, non-neural mechanisms maintain the constriction. A key role in these events has been attributed to the intracellular signaling factors RhoA and Rho kinase (RhoA/ROCK) [7]. They are activated by mitochondrial superoxide, the production of which is augmented by direct cutaneous vessel cooling. Activated RhoA/ROCK stimulates vasoconstriction by increasing the net phosphorylation state of MLC by increasing MLCK activity while simultaneously decreasing MLC phosphatase activity [7]. (Some recent evidence suggests that the cutaneous vasoconstrictor response to whole body cooling may also be partially mediated by the ROCK pathway [8].)

The diminished vasoconstrictor response to whole body cooling that occurs with aging is, however, not paralleled by a similarly reduced responsiveness upon localized skin cooling. Although some changes occur with age in the intracellular processes underlying the RhoA/ ROCK pathway, the net result is not different so that the magnitude of the vasoconstrictor response to local cooling is unaffected [7].

\section{Heat Exposure}

A rise in skin and core temperatures such as caused by whole body heat exposure induces cutaneous vasodilation in glabrous skin by the withdrawal of the extant basal sympathetic tone ('passive vasodilation'). In nonglabrous skin, if core temperature continues to rise, sympathetic cholinergic nerves additionally become activated, releasing acetylcholine (ACh). In addition to driving the sweating response by its action on eccrine sweat gland muscarinic $M_{1}$ and $M_{3}$ receptors, this neurotransmitter contributes to a further, early increase in skin blood flow by stimulating the production of the second messenger nitric oxide (NO). (It should be noted here that, although virtually coincident responses to heat exposure, sweating and active vasodilation are not causally related.) Blood flow is next augmented actively ('active vasodilation') by one or more as yet unidentified transmitters that are coreleased with $\mathrm{ACh}$ and then supplant its action $[4,5]$. These putatively include vasoactive intestinal peptide (VIP), substance P (SP), calcitonin gene-related peptide (CGRP), histamine, and neurokinin 1 [4]; their order, interrelationships and mechanisms of action in the process are still unclear. Prostaglandins have lately also been implicated in active cutaneous vasodilation [9].

The reflex cutaneous vasodilator response to heat exposure is attenuated in the aged. Both a delay in the initiation of the response and a reduced maximal vasodilator capacity have been documented $[7,10]$. These can, however, be reversed by acute treatment with antioxidants or inhibition of arginase (thereby maintaining normal levels of arginine, the substrate for NO synthase [NOS]), suggesting that the age-related accumulation of ROS mentioned earlier may limit NOS function, i.e. impair NO-dependent VSM relaxation.

Direct skin heating causes an initial, abrupt, transient increase in local blood flow mediated by noxious-heatsensing transient receptor potential vanilloid 1 (TRPV1)expressing neurons, and a consequent axon reflex. This is followed by a further rise to a plateau, due to the release 
of NO; $\mathrm{NO}$ in this context also inhibits the extant noradrenergic activity, enhancing the vasodilation. Eventually, the dilation slowly abates, but it is not clear whether this is due to a reduction in the vasodilator function or a recovery of the vasoconstrictor tone [5].

In the elderly, the initial vasodilator response to local heating evoked by stimulation of temperature-sensitive nociceptors and its attendant axon reflex is reduced. The ensuing NO-mediated response is similarly depressed as is the maximal skin blood flow, due essentially to the same factors that limit the vasodilator response to whole body heating [7]. It would thus appear that underlying the depressed skin blood flow response of elderly subjects to heating, whether whole body or localized, are a gradual, age-related decrease in NO synthesis (probably due to decreased L-arginine bioavailability) on the one hand and an increase in NO degradation (due to ROS accumulation) on the other. All these factors combine to limit the ability of aged subjects to lose heat in a warm environment, thereby causing their core temperature to rise and predisposing them to hyperthermia.

\section{Cardiovascular Integration}

The adaptive responses of cutaneous blood flow to whole body heating and cooling, however, do not happen in isolation. Rather, they are parts of an integrated cardiovascular response to these stimuli designed to insure the maintenance of blood pressure and the proper, vital distribution of the cardiac output. Thus, in the heat, the increased conductance of the skin is balanced by a decreased blood supply to the splanchnic region and the kidneys and supplemented by an increase in the cardiac output. This response pattern is mediated by the excitation of the sympathetic nerves to these beds.

In aged heat-exposed subjects, the increase in cardiac output is significantly smaller than in adults, probably due to the already mentioned, age-related, general decreased sensitivity of adrenoceptors, in this case the weakened $\beta$ receptors of the heart limiting the magnitude of the stroke volume. There is also some evidence that less than the combined splanchnic and renal blood flows may be redirected to the skin. This could be due to the reported age-related selective decrease in the tonic discharge rate of visceral sympathetics [11]. A similar impairment may account for the smaller increase in cardiac output of the elderly during cold exposure. On the other hand, the blood pressure increases more in the elderly than in the adult, particularly upon abrupt transition from thermoneutrality into cold, but this effect has been attributed to an age-associated rise in central arterial stiffness.
Also contributing to the lower skin temperature of the elderly and their impaired ability to conserve heat in a cold environment or, conversely, to limit heat influx in a warm environment is age-related thinning of the dermal layer of nonglabrous skin, implying less vascularization [12]. The subcutaneous fat layer is also reduced with ageing while the VSM itself hypertrophies over time.

\section{Sweating}

As described earlier, heat dissipation in an environment warmer than the skin temperature (or whenever heat loss by conduction, convection and radiation is inadequate) depends on the evaporation of sweat. The magnitude of sweating differs from one region of the skin to another due to differences in the regional distribution of eccrine sweat glands and their specific density in a given site. The sweat output per gland also varies, depending on many factors [13]. With aging, both the total body and the local sweat rate responses to passive heating are decreased. However, the latter does not occur uniformly all over the body. Rather, it would appear that the decrement proceeds sequentially from the lower limbs to the back of the upper body, then to the front of the upper body, thereafter to the upper limbs, and finally to the forehead [14]. The data further indicate that this is due to a decrease in individual sweat gland output rather than in the number of actively secreting glands, but it is still uncertain whether sweat gland structural changes (e.g. atrophy) or reduced cholinergic sensitivity is responsible for the depressed response. Nevertheless, as aging progresses, the number of sweat glands activated by heat decreases as well. Although, as mentioned earlier, sweat rate and skin blood flow are regulated separately, the production of sweat depends on an adequate supply of blood (the source of its liquid), so that hypo- or dehydration, a frequent condition of senescent individuals, is a contributing factor to the risk of hyperthermia in the elderly.

\section{Heat Production}

As mentioned at the beginning of this review, heat is constantly generated by the body as a waste product of its constant metabolic activity. It is what accounts for its temperature and must constantly be dissipated to prevent hyperthermia. But when the loss of heat exceeds its production, threatening hypothermia (as during cold exposure), more heat must be produced by the body. The prin- 
cipal source of this extra heat is the skeletal muscle mass. Shivering thermogenesis in skeletal muscle is estimated to provide up to one-third of the total heat production of cold-exposed adults [15]. The amount of this contribution declines with advancing age [16]. It has been attributed to the progressive atrophy of the skeletal muscle mass, but because physical exercise maintains or even increases lean body mass and reduces fat weight, it is questioned whether this atrophy reflects biological aging or a sedentary lifestyle. Similarly, various changes in the glucose metabolism of skeletal muscle, e.g. impaired glucose uptake, increased insulin resistance, and decreased glucose tolerance, that occur with age and would reduce quantitatively shivering thermogenesis have now also been attributed, in part, to inactivity and obesity [16]. There is also no clear evidence that lipid metabolism, the delivery of substrates, and the activity of enzymes involved in aerobic metabolism are altered by age in, particularly, weight-bearing muscles during shivering. It would thus appear that, although a decrease in shivering capacity is associated with aging, it is more likely due to the customarily sedentary lifestyle of older people than to biological aging per se.

Shivering thermogenesis is not, however, the only means of cold-induced heat production. Another mechanism exists that is not dependent on skeletal muscle contractions, but rather on the metabolic activity of a specialized tissue, brown adipose tissue (BAT) nonshivering thermogenesis (NST) [16-18]. Abundant deposits of this tissue are strategically located around the vital organs of, particularly, newborn animals, including human, and adult rodents whose skeletal muscle mass is relatively small and body volume (i.e. weight)-to-surface area ratio is high. In a cold environment, its activation can readily double the basal rate of heat production. This is accomplished by the release of NE and its binding to $\beta_{3}$-adrenoceptors in the plasma membrane of brown adipocytes, which activates adenylcyclase and yields adenosine $3^{\prime}, 5^{\prime}$ cyclic monophosphate (cAMP). This initiates the hydrolysis of triglycerides from intracellular lipid droplets, causing the release of free fatty acids and their oxidation in the mitochondria. But in this case, the oxidation is uncoupled from the electron transfer system by an uncoupling protein, $\mathrm{UCP}_{1}$ (also called thermogenin) in the mitochondrial inner membrane, so that heat rather than ATP is the end product of the respiratory chain [16-18]. Other substrates are derived from the circulation and also combusted. BAT, however, has generally been reported to atrophy and disappear over the first few months of life so that its role in adult thermogenesis is thought to be minor at best. But, thanks to new technologies, recent, more meticulous searches have uncovered significant amounts of this tissue still present (or perhaps regenerated) in adults $[19,20]$. Its activation, therefore, could account for a significant proportion of the extra heat produced on cold exposure. If so, a reduction of this tissue's capacity to produce heat could explain the elderly's reduced thermogenic response to cold exposure. But it remains to be established whether the progressive atrophy of the mass of BAT and/or the diminution of its metabolic capacity represents a normal aging process or is a consequence of its disuse due to compensatory adjustments by the individual to keep warm by, for example, behavioral means.

To the extent that an increase in heat production connotes an increase in the rate of $\mathrm{O}_{2}$ consumption, it follows that the effectiveness of this process depends on an adequately functioning cardio-respiratory system. Hence, age-related cardiac or pulmonary dysfunctions could impair the ability of afflicted subjects to generate sufficient extra heat in a cold environment. By the same token, to the extent that thermogenic mechanisms are supported at different levels by various hormones (e.g. epinephrine, thyroxine, cortisone), age-associated endocrine deficiencies could similarly affect thermogenic responsiveness.

\section{Thermosensitivity}

The initiation of the thermoregulatory responses to cold and heat exposure described above would not occur without alerting signals informing the central thermoregulatory controller in the brain that appropriate defense measures must be quickly instituted. These signals are provided by thermosensors located both in the periphery and the core of the body. Free nerve endings constitute warm and cold sensitive receptors (thermoTRPs) that lie just below the epidermis and, when stimulated, send their signals via spinal pathways to the preopticanterior hypothalamus, where they are integrated and transduced into new signals to the thermoeffector organs. It is not necessary in the present context to describe in detail the anatomy and physiology of this system; interested readers are referred to other reviews on this subject [21]. Suffice it to note that age significantly alters the structures and functions of the nervous system so that age-related changes in thermal sensitivity and perception may be anticipated. Indeed, thermal sensitivity decreases with age [22]. Both warm and cold sensitivities decline, but the decrement in the perception of warmth is more 
pronounced than that of cold; interestingly however, the sensation of heat-pain is preserved. The reduction in thermosensitivity follows a distal-proximal pattern, i.e. it occurs earlier in the lower than in the upper extremities and in the core [14]. Causes that have been attributed for this loss include age-related structural changes in the skin and/or its microvascular network (i.e. $\mathrm{O}_{2}$ supply) that could directly affect the functionality of the thermosensitive free nerve endings. Alterations in the nerve density and electrophysiological and neurochemical properties of the afferent pathway to the brain, i.e. the primary afferent fibers, the secondary and tertiary neurons in the spinal cord, and the brain itself, have also been implicated. To date, peripheral fiber loss and lower conduction velocity have been documented as two important factors in the decreased thermosensitivity of the elderly. The expressions of the voltage-dependent sodium channel Nav 1.8 and the thermosensitive cation channel TRPV1 have also been found to be reduced in the dorsal root ganglion and peripheral nerves of aged mice [23]; such a reduction would impair the efficient transport of membrane channel proteins (that regulate ion flow in response to stimuli) to the peripheral afferents. This reduction in expression is more prominent in neurons at lumbar levels that project to the lower limbs, consistent with the distalproximal direction of thermosensitivity loss mentioned above.

\section{Behavior}

Although the autonomic responses to cold and heat exposure described above are clearly appropriate and effective, they are nevertheless limited because they often are insufficient to prevent hypo- or hyperthermia. Nature therefore has provided another protective mechanism, behavioral thermoregulation. Indeed, as mentioned in the introduction, behavioral responses are most often initiated before autonomic responses. They occur as soon as a change in ambient temperature is sensed by the cutaneous thermosensors or perceived via other sensory organs, e.g. the eyes - looking at a thermometer -, entraining activities such as adding or removing clothing, turning on or off heating or cooling systems, etc. There are few studies on the age-related changes in behavioral thermoregulation in humans, but the general impression is that the elderly are less competent in this respect than adults. How much of this may be due, for instance, to subjective thermal perception, incomprehension of the risk, individual physical handicaps, weaken- ing of peripheral thermosensitivity, or other deficits remains to be studied. There is some evidence that older people may prefer warmer ambient temperatures, perhaps as a compensation for their failing autonomic responsiveness to the generally cooler environment in which humans live [24]. More data are available on the effect of age on the behavioral thermoregulation of animals, and the interested reader is referred to them [25].

\section{Fever}

The thermoeffector mechanisms that underlie the production of fever are the same as those that are activated in response to cold exposure. Therefore, the deficiencies that attend the elderly's defense of their core temperature in a cold environment also afflict their ability to adequately raise core temperature in defense against infectious challenges. Indeed, fever (as defined as an oral temperature $\geq 37.8^{\circ} \mathrm{C} / 100^{\circ} \mathrm{F}$ ) is often absent in benign infections and lower in severe infections (e.g. meningitis, pneumonia) of approximately $25 \%$ of geriatric as compared with younger patients [26]. The effect, however, of age-related thermoeffector deficits on the robustness of the febrile response may be somewhat overestimated. Since, as discussed earlier, the basal core temperature of the aged tends to be lower than that of younger individuals, the fever of the former could likewise be proportionally lower, i.e. not attain its classic height, even when the temperature rise provoked by their infection is of similar magnitude as that in younger patients. In fact, lowering the fever threshold to (oral) $37.2^{\circ} \mathrm{C} / 99^{\circ} \mathrm{F}$ (or, alternatively, setting it at $1.1^{\circ} \mathrm{C} / 2^{\circ} \mathrm{F}$ above baseline) greatly increases the reported incidence of infectious fever in the aged [25]. More determinative of the weaker fevers of the aged are demonstrated alterations on the affector innate immune side of the host response to infection, e.g. decreased production of pro-inflammatory (pyrogenic) mediators such as interleukin-1 and tumor necrosis factor; but these changes are beyond the scope of this review. In any case, the blunting of the febrile response is a serious detriment insofar as it may retard the recognition of infections in aged patients on the one hand and diminish the benefits of an elevated body temperature on host defense on the other. 


\section{References}

$>1$ Kelly G: Body temperature variability. 1. A review of the history of body temperature and its variability due to site selection, biological rhythms, fitness, and aging. Altern Med Rev 2006;11:278-293.

$\checkmark 2$ Lu SH, Lessure AR, Dai YT: A systematic review of body temperature variations in older people. J Clin Nurs 2010;19:4-16.

$>3$ Waalen J, Buxbaum JN: Is older colder or colder older? The association of age with body temperature in 18,630 individuals. J Gerontol A Biol Sci Med Sci 2011;66:487492.

4 Charkoudian N: Mechanisms and modifiers of reflex induced cutaneous vasodilation and vasoconstriction in humans. J Appl Physiol 2010;109:1221-1228.

$\checkmark 5$ Johnson JM, Kellogg DL Jr: Mechanisms of thermoregulatory and thermal control in the cutaneous circulation. Front Biosci 2010;S2: 825-853.

$\checkmark 6$ Holowatz LA, Kenney WL: Peripheral mechanisms of thermoregulatory control of skin blood flow in aged humans. J Appl Physiol 2010;109:1538-1544.

7 Holowatz LA, Thompson-Torgerson C, Kenney WL: Aging and the control of human skin blood flow. Front Biosci 2010;15:718739.

$>8$ Lang JA, Jennings JD, Holowatz LA, Kenney WL: Reflex vasoconstriction in aged human skin increasingly relies on Rho kinase-dependent mechanisms during whole body cooling. Am J Physiol Heart Circ Physiol 2009;297:H1792-H1797.
9 McCord GR, Cracowski JL, Minson CT: Prostanoids contribute to cutaneous active vasodilation in humans. Am J Physiol Regul Integr Comp Physiol 2006;291:R596-R602.

10 Holowatz LA, Thomson-Torgerson C, Kenney WL: Altered mechanisms of vasodilation in aged human skin. Exerc Sport Sci Rev 2007;35:119-125

11 Helwig BG, Parimi S, Ganta CK, Cober R, Fels RJ, Kenney WL: Aging alters regulation of visceral sympathetic nerve response to acute hypothermia. Am J Physiol Regul Integr Comp Physiol 2006;291:R573-R579.

12 Petrofsky JS, McLellan K, Bains GS, Prowse M, Ethijaru G, Lee S, Gunda S, Lohman E III, Schwab E: The influence of ageing on the ability of the skin to dissipate heat. Med Sci Monit 2009;15:CR261-CR268.

13 Shibasaki M, Crandall CG: Mechanisms and controllers of eccrine sweating in humans. Front Biosci 2010;S2:685-696.

14 Inoue Y, Kuwahara T, Araki T: Maturationand aging-related changes in heat loss effector function. J Physiol Anthropol Appl Human Sci 2004;23:289-294.

15 Haman F, Blondin DP, Imbeault MA, Maneshi A: Metabolic requirements of shivering humans. Front Biosci 2010;S2:11551168.

16 Florez-Duquet M, McDonald RB: Cold-induced thermoregulation and biological aging. Physiol Rev 1998;78:339-358.

17 Silva EJ: Physiological importance and control of non-shivering facultative thermogenesis. Front Biosci 2011;S3:352-371.

18 Richard D, Picard F: Brown fat biology and thermogenesis. Front Biosci 2011;16:12331260 .
19 Nedergaard J, Cannon B: The changed metabolic world with human brown adipose tissue: Therapeutic visions. Cell Metab 2010;11: 268-272.

20 Saely CH, Geiger K, Drexel H: Brown versus white adipose tissue: a mini-review. Gerontology DOI: 10.1159/000321319.

-21 Bandell M, Macpherson LJ, Patapoutian A: From chills to chilis: mechanisms for thermosensation and chemesthesis via thermoTRPs. Curr Opin Neurobiol 2007;17:490497.

22 Guergova S, Dufour A: Thermal sensitivity in the elderly: a review. Ageing Res Rev 2011; 10:80-92.

23 Wang S, Davis BM, Zwick M, Waxman SG, Albers KM: Reduced thermal sensitivity and Nav 1.8 and TRPV1 channel expression in sensory neurons in aged mice. Neurobiol Aging 2006;27:895-903.

24 Van Hoof J, Mazej M, Hensen JLM: Thermal comfort: research and practice. Front Biosci 2010;15:765-788.

25 Terrien J, Perret M, Aujard F: Behavioral thermoregulation in mammals: a review. Front Biosci 2011;16:1428-1444.

26 High KP, Bradley SF, Gravenstein S, Mehr DR, Quagliarello VJ, Richards C, Yoshikawa TT: Clinical practice guidelines for the evaluation of fever and infection in older adult residents of long-term care facilities: 2008 update by the Infectious Diseases Society of America. Clin Infect Dis 2009;48:149-171. 